\title{
Imagery instructions improve memory in blind subjects
}

\author{
JOHN JONIDES, ROBERT KAHN, and PAUL ROZIN \\ University of Pennsylvania, Philadelphia, Pennsylvania 19174
}

\begin{abstract}
Instructing subjects to make images of word pairs greatly improves their memory for these pairs. It is commonly assumed that the memory improvement results from some advantage in memory of visual images over words. We show that the typical improvement in memory with imagery instructions that occurs in normal subjects also occurs in congenitally and totally blind adults. Hence, the mnemonic imagery effect cannot be explained with reliance on a mechanism that specifically relies on vision. We also demonstrate that the results cannot be explained in terms of imagery in nonvisual modalities.
\end{abstract}

The effectiveness of imagery instructions in improving paired-associate learning has been known since the time of Simonides 2500 years ago (Yates, 1966) and has been confirmed many times in recent years (e.g., Bugelski, 1968; Bugelski, Kidd, \& Segmen, 1968; Paivio, 1969). Why do imagery instructions have so dramatic an effect? Although several factors have some effect on recall performance [among them, the figural unity (Atwood, 1971; Bower, 1970) and plausibility (Collyer, Jonides, \& Bevan, 1972) of the images], the basic nature of the enhancement produced by imagery instructions remains obscure. Introspective reports by subjects strongly implicate vision; for example, given the instruction to image the pair "horse-rock," many subjects report that they "see" (in their mind's eye) a horse or a team of horses dragging a huge rock. How seriously should such introspection be taken in constructing a reasonable model accounting for the mnemonic effectiveness of imagery instructions? To what extent are "images" involved, and to what extent are such "images" visual?

Results from several quarters support the importance of the visual aspect of the image in producing impressive memory performance. Some evidence suggests that visual memory is superior to verbal memory on recognition tasks (Shepard, 1967; Standing, Conezio, \& Haber, 1970). Furthermore, Atwood (1971) has shown that visually distracting stimuli introduced during original presentation of the word pairs are more detrimental to later recall under imagery instructions than are auditory distracting stimuli. The reverse was true with verbal instructions not involving imagery. Finally, given imagery instructions, words with high

This experiment was conceived in a General Honors Psychology I course at the University of Pennsylvania taught by Paul Rozin and John Jonides in the fall of 1972. Robert Kahn was a member of that class who subsequently ran the experiment reported here under the supervision of J. J. and $P$. R. We gratefully acknowledge the helpful discussions with the class members in designing a pilot experiment (run by Cynthia Dell) which preceded this one. We also acknowledge the helpful criticism of an earlier manuscript by James Johnston, James McClelland, and Deborah Macmillan. This research was supported by a Sigma Xi grant to J. J. and an NIH physiological psychology training grant to the University of Pennsylvania. J. J. is a trainee under this grant. The authors are listed in alphabetical order. Reprint requests should be sent to Department of Psychology, University of Pennsylvania, 3815 Walnut Street, Philadelphia, Pennsylvania 19174. visual imageability (as independently rated by subjects) produce recall superior to those with low visual imageability (Paivio, 1969).

The critical test of the hypothesis that the images must be visual, however, is to expose blind individuals to the imagery instructed paired-associate task. Although some previous investigators have used blind subjects in tasks of this type, none have used imagery instructions with totally and congenitally blind individuals. Craig (1973) has demonstrated superior memory performance by blind subjects on lists composed of highly imageable as opposed to not easily imageable words. The relevance of this finding to the effect of imagery instructions on blind individuals is not obvious, although there undoubtedly is some relation. In addition, Craig used free recall of single word lists (not the typical paired-associate cued recall task) and included partially sighted and noncongenitally blind subjects in his sample. Another study by Paivio and Okovita (1971) is more comparable to the present one in the use of a paired-associate task and the use of both congenitally and totally blind subjects. However, this study again involved the manipulation of rated word imageability, and not the use of instructions to form images. In the present study, memory performance of normal and congenitally blind subjects was compared under either "standard" or imagery instructions.

\section{METHOD}

Subjects were 16 congenitally and totally blind college students from Goucher College, Johns Hopkins University, and the University of Maryland, and 16 normally sighted college students from the University of Pennsylvania. Each subject in both groups served in two conditions. In the first, a list of 20 noun pairs was read aloud at the rate of one pair every $10 \mathrm{sec}$. Each subject was told that he should remember the pairs as best he could because he would be tested for recall of the second noun in each pair, given the first as a cue. The second condition involved another list of 20 noun pairs, but each subject was told to remember the list by imagining a relationship between the words in each pair. As an example, each subject was given the pair locomotive-dishtowel. The experimenter suggested that an image of this might be a dishtowel wrapped around a locomotive. In both conditions testing immediately followed one 


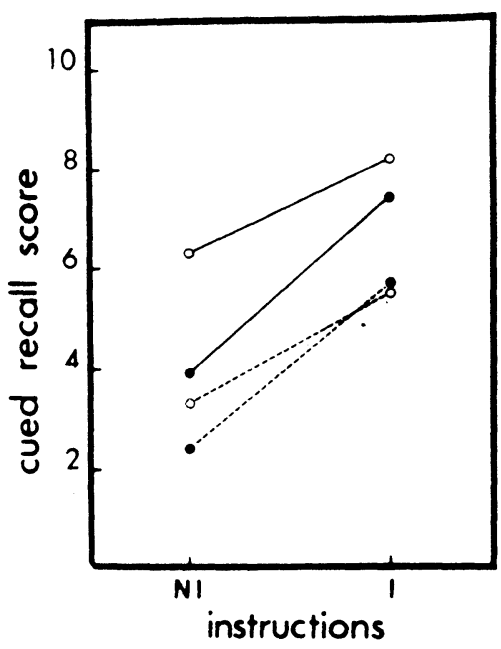

Figure 1. Mean performance of normal and congenitally blind subjects on paired-associates, under no-imagery (NI) or imagery (I) instructions. Filled circles represent mean data from 16 blind subjects, open circles form 16 normals. Solid lines represent data from highly imageable word pairs, dotted lines from word pairs that are not easily imageable.

presentation of the list and involved cuing subjects verbally with the first noun of each pair in turn, and requiring recall of the second noun. Blind subjects typed their responses and normally sighted students wrote them out. All subjects were allowed $10 \mathrm{sec}$ to respond before the experimenter proceeded to the next cue in the list.

The lists were constructed so that 10 pairs in each were comprised of nouns rated high in imageability, while the other 10 pairs of nouns were rated low on this scale. All but 12 of the 80 words used in the experiment were chose from a list due to Paivio, Yuille, and Madigan (1968). The mean imageability ratings with their standard deviations for both types of words on each list are: List 1 -high $\mathrm{I}=6.54, \mathrm{SD}=.26$; List 1 -low $\mathrm{I}=3.29, \mathrm{SD}$ .76 ; List 2-high $\mathrm{I}=6.55, \mathrm{SD}=.15 ;$ List 2-low $\mathrm{I}=3.54, \mathrm{SD}=.89$ (all ratings are based on a scale of 1-7). Examples of some pairs used in the experiment are nail-orange and elephant-bridge (rated high on imageability), and situation-glory and custom-affection (rated low). These high and low imageable pairs were mixed randomly within each list. Half the subjects in each group were given List 1 followed by List 2 , and half, the reverse (with imagery instructions always given with the second list). A comparison of the recall scores indicated that the lists did not differ in difficulty either with no-imagery or imagery instructions: $\mathrm{I}: \mathrm{t}(30)=.83, \mathrm{p}>.20 ; \mathrm{NI}: \mathrm{T}(30)=1.02, \mathrm{p}>.10$.

\section{RESULTS AND DISCUSSION}

It is clear from Figure 1 that overall, blind subjects perform more poorly than sighted ones $(F=4.59, \mathrm{df}=$ $1 / 30, p<.05)$. However, the important point is that both subject groups show a marked improvement with imagery instructions, averaging $51 \%$ [effect of instructions as revealed by analysis of variance: $F=$ $105.4 ; \mathrm{df}=1 / 30 ; \mathrm{p}<.001]$. Furthermore, this improvement seems to be of the same order of magnitude for the blind group as for the sighted group. ${ }^{1}$ Surprisingly then, we must conclude that imagery instructions have substantial mnemonic value in the absence of vision.

It might be noted that the design of this experiment did not allow us to exclude the possibility that the improvement of subjects on the second list was due to a simple practice effect. We subsequently ran a group of 23 University of Pennsylvania undergraduate students who were given the two experimental lists to remember under no-imagery instructions for both lists. The experimental conditions were exactly as in the main experiment. An analysis of variance performed on these control data revealed no hint of an overall improvement from the first to the second list $(F=.35$; $\mathrm{df}=1 / 22$; $\mathrm{p}>.5)$. Furthermore, there was no significant interaction of material type (high vs. low imageable) with list $\operatorname{order}(\mathrm{F}=.87 ; \mathrm{df}=1 / 22 ; \mathrm{p}=.36)$. Finally, a group of 13 subjects with imagery instructions on both lists was run. Again, no significant improvement with practice was found, nor was there any interaction of material type with list order $(F=.04, \mathrm{df}=1 / 12 ; \mathrm{p}>.5$; $\mathrm{F}=1.43 ; \mathrm{df}=1 / 12 ; \mathrm{p}=.25$, respectively). These two control experiments adequately demonstrate that the improvement from List 1 to List 2 in the main experiment is not due to practice, but rather is a function of the instructional manipulation.

It is conceivable that equally effective "images" can exist in sense modalities other than vision. Under this hypothesis, blind subjects rely on images that are auditory, tactile, etc. To address this possibility, we had the blind and sighted subjects rate each highly imageable word in the two lists ( 40 words in all) for visual, auditory, and tactile imageability. A modality specific imagery hypothesis would predict some significant correlation between at least one of these ratings and recall scores. We found no such significant correlations (the highest correlation was .42). Moreover, certain word pairs which have no obvious nonvisual imageability were recalled as well by blind subjects as pairs rated high in nonvisual imageability (e.g., cottage-mountain vs. tweezers-ball). Another suggestive bit of evidence (Figure 1) is that imagery instructions produce equal improvement on both high- and low-imageable words (supported by the lack of a significant interaction between instructions and type of stimulus material: $\mathrm{F}<1.0$ ). These findings discredit an hypothesis based on modality specific images.

Two alternative explanations of the mnemonic imagery effect have been suggested. The first, proposed by Neisser (1972), focuses on a mental representation of spatial interaction as the critical variable. The spatial representation is conceived as being more abstract than a particular sensory modality, and certainly not vision dependent. Although this notion can account for the blindness data, it cannot easily explain the enhanced effect of imagery instructions on abstract (low-imageable) words. 
The second view takes the imagery effect completely out of the realm of images, introspections notwithstanding. The effects of the instructions can be viewed simply as promoting the establishment of a meaningful relationship between two words which should enhance their memorability (see, e.g., Bower, 1972).

We are quite sure that the imagery effect is not essentially visual. Frankly, we are quite puzzled as to what it is.

\section{REFERENCES}

Atwood, G. An experimental study of visual imagination and memory. Cognitive Psychology, 1971, 2, 290-299.

Bower, G. H. Analysis of a mnemonic device. American Scientist, 1970, 58, 496-510.

Bower, G. H. Mental imagery and associative learning. In L. W. Gregg (Ed.), Cognition in learning and memory. New York: Wiley, 1972, Pp. 51-88.

Bugelski, B. R. Images as mediators in one-trial paired associates learning: II. Self-timing in successive lists. Journal of Experimental Psychology, 1968, 77, 328-334.

Bugelski, B. R., Kidd, E., \& Segmen, J. Image as a mediator in one-trial paired associate learning. Journal of Experimental Psychology, 1968, 76, 69-73.

Collyer, S. C., Jonides, J., \& Bevan, W. Images as memory aids: Is bizarreness helpful? American Journal of Psychology, 1972, 85, 31-38.

Craig, E. M. Role of mental imagery in free recall of deaf, blind, and normal subjects. Journal of Experimental Psychology, $1973,97,249-253$.
Neisser, U. Changing conceptions of imagery. In P. W. Sheehan (Ed.). The function and nature of imagery. New York: Academic Press, 1972, 234-249.

Paivio, A. Mental Imagery in associative learning and memory. Psychological Review, 1969, 76, 241-263.

Paivio, A., \& Okovita, H. W. Word imagery modalities and associative learning in blind and sighted subjects. Journal of Verbal Learning and Verbal Behavior, 1971, 10, 506-510.

Paivio, A., Yuille, J. C., \& Madigan, S. A. Concreteness, imagery and meaningfulness values for 925 nouns. Journal of Experimental Psychology, 1968, 76, 1, Part 2.

Shepard, R. N. Recognition memory for words, sentences, and pictures. Journal of Verbal Learning and Verbal Behaviour, $1967,6,156-163$.

Standing, L., Conezio, J., \& Haber, R. N. Perception and memory for pictures: Single-trial learning of 2500 visual stimuli. Psy chonomic Science, 1970, 19, 73-74.

Yates, F. The art of memory. Chicago: University of Chicago Press, 1966.

\section{NOTE}

1. Figure 1 suggests that blind subjects may improve even more with I instructions than sighted subjects. In fact this interaction is statistically significant $(F=5.36 ; \mathrm{df}=1 / 30$; $p<.05$ ). However, this effect may be somewhat misleading since there is a slight ceiling effect which tends to attenuate the improvement of the sighted group (a slightly greater number of sighted subjects, 4 out of 16, than blind subjects, 2 out of 16 , had a perfect recall score for the highly imageable words under $I$ instructions). The ceiling difference is small, but might account for the slightly greater improvement of the blind group. expecially considering that the blind subjects have lower scores than the sighted in the NI condition.

(Received for publication January 10, 1975.) 